\title{
Evaluación del aumento de peso en lechones durante la lactancia en parideras tecnificadas y tradicionales
}

\author{
María Johana García-Aguirre ${ }^{1}$ \\ Rigoberto Villa-Ramírez ${ }^{2}$ \\ Jaime Hurtado-Villegas ${ }^{3}$
}

Fecha de recepción: 16 de febrero de 2019

Fecha de aceptación: 24 de mayo de 2019

\section{Resumen}

El objetivo de este trabajo fue evaluar el aumento de peso en lechones durante la lactancia en parideras tecnificadas y tradicionales. La investigación se realizó en las instalaciones de la Granja Experimental Bengala de la Universidad del Quindío, ubicada en el municipio de Filandia, a 2050 metros de altitud, donde se estimó la ganancia de peso de 53 lechones de cerdas landrace, alojados en parideras tradicionales y tecnificadas con planchas de calentamiento previamente desinfectadas. Durante la etapa de destete (21 días), el manejo para ambos tratamientos fue semejante, lo que implicó manipular la temperatura dentro de las parideras para mantener el calor corporal de los neonatos porcinos. Se registraron los pesos desde su nacimiento hasta terminar la etapa de destete, se empleó un diseño completamente al azar. El análisis de varianza mostró que ningún valor-P es menor que 0,05 ; por tanto, ninguno de los factores tiene un efecto estadísticamente significativo sobre el peso con un $95.0 \%$ de nivel de confianza. El promedio general desde el nacimiento hasta el destete (21días) en parideras tecnificadas está en 6400 g/lechón, lo que indica que el aumento semanal de los lechones en jaulas tecnificadas se encuentra en un promedio de $1300 \mathrm{~g}$, mientras que el aumento de los lechones nacidos en parideras no tecnificadas o tradicionales es, en promedio, de $1200 \mathrm{~g}$ semanales.

Palabras clave: celdas de parición; destete; lechón; peso al destete.

\section{Evaluation of Weight Gain in Piglets during Lactation in Technical and Traditional Farrowing Rooms}

\section{Abstract}

The objective of this work was to evaluate the weight gain in piglets during lactation in technified and traditional farrowing rooms. This research was carried out in the facilities of the Bengal Experimental Farm of the University of Quindío, located in the municipality of Filandia at 2,050 meters of altitude, where the weight gains of 53 piglets of landrace sows were evaluated, which were housed in traditional and

\footnotetext{
${ }^{1}$ Universidad de Quindío (Armenia, Colombia). mgarcia@uqvirtual.edu.co. ORCID: 0000-0002-7871-3380.

${ }_{2}^{2}$ M. Sc. Universidad de Quindío (Armenia, Colombia). rivilla@uniquindio.edu.co. ORCID: 0000-0002-4355-2230.

${ }^{3}$ M. Sc. Universidad de Quindío (Armenia, Colombia). jhurtadov@uniquindio.edu.co. ORCID: : 0000-0002-3625-1763. 
technified farrowing rooms with previously disinfected heating plates. During the weaning stage (21 days), the handling for both treatments was similar, which implied manipulating the temperature inside the farrowing's to maintain the body heat of the swine neonates. The weights were recorded from birth to the end of the stage of weaning; a completely random design was used. The analysis of variance showed that no P-value is less than 0.05 ; therefore, none of the factors has a statistically significant effect on weight with a $95.0 \%$ confidence level. The general average from birth to weaning (21 days) in technified farrowing rooms is $6400 \mathrm{~g} /$ piglet, which indicates that the weekly increase of piglets in technified cages is at an average of $1300 \mathrm{~g}$; while piglets born in non-technified or traditional farrowing rooms earn an average of $1200 \mathrm{~g}$ per week.

Keywords: farrowing pens; piglets; weaning; weaning weight.

Para citar este artículo:

García-Aguirre, M. J., Villa-Ramírez, R., \& Hurtado-Villegas, J. (2019). Evaluación del aumento de peso en lechones durante la lactancia en parideras tecnificadas y tradicionales. Ciencia y Agricultura, 16(3), 7-16. https://doi.org/10.19053/01228420.v16.n3.2019.9513.

Esta obra está bajo licencia internacional Creative Commons Reconocimiento 4.0 


\section{INTRODUCCIÓN}

La producción porcícola en Colombia ha empezado a evolucionar con el fin de perfeccionar la calidad y facilitar la producción a los productores. Los nuevos sistemas de producción tienen grandes ventajas, porque le brindan a la cerda y a sus lechones un mayor bienestar animal en cuanto a temperatura e higiene (Asociación Colombiana de Porcicultores [Porcicol], 2009).

Durante los últimos años se han desarrollado complejas técnicas de alojamientos para las diferentes etapas de producción de cerdos y se han obtenido unas buenas técnicas productivas, donde el logro primordial es destetar el mayor número de lechones (Piñero \& Montalvo, 2015).

Mientras las cerdas están embarazadas pueden tener diferentes sistemas de manejo y de alojamientos según sus exigencias. Los dos tipos de alojamiento conocidos son en confinamiento (jaula) o con independencia (corral), uno y otro se diferencian por el área conferida a cada animal, y tienen ventajas y desventajas dependiendo de los días de gestación de la cerda, pero sea cual sea el tipo de instalación se deben dar las mejores condiciones posibles de comodidad y confort (Brunori \& Figueroa, 2012).

De acuerdo con Manteca (2011), las jaulas o parideras tecnificadas tienen grandes ventajas, ya que ofrecen un sitio adecuado tanto para la cerda como para sus lechones; además cuentan con una plancha térmica que va regulando la temperatura a medida que los lechones van creciendo. La función de esta plancha es mantener a los lechones bajo condiciones térmicas adecuadas. La jaula tecnificada tiene buenos canales de drenaje que permiten la correcta disposición de los desechos sólidos y líquidos resultantes. Este aspecto, además de reducir el impacto medioambiental, garantiza mejores condiciones sanitarias para los animales allí alojados.

Las parideras tradicionales presentan algunas dificultades: en primer lugar, no cuentan con un sitio adecuado para materiales, estructura, medidas de densidad e instalaciones óptimas para la cerda y sus lechones; y, en segundo lugar, permanecen en condiciones ambientales inapropiadas, tales como humedad, temperatura, ventilación e iluminación, que afectan directamente el entorno ambiental y el desarrollo normal del periodo de lactancia en las diferentes producciones porcícolas.

Según Beltrán (2010), los lechones son los cerdos recién nacidos que permanecen con su madre tomando leche hasta el destete. Al nacer pesan entre 1 y $1,5 \mathrm{~kg}$ y son muy susceptibles a las enfermedades, por los cambios de temperatura y las corrientes de aire, por lo que normalmente durante la lactancia muere mínimo el 10 $\%$ de ellos.

La paridera es un corral especial, adecuado para alojar a la cerda y sus lechones. Tiene que ser un lugar libre de humedad, con una temperatura ambiente de 20 a 25 
${ }^{\circ} \mathrm{C}$, sin corriente de aire, con una construcción resistente, que garantice durante las 72 horas posteriores al parto la protección a los recién nacidos, pues los lechones no son lo suficientemente fuertes para impedir ser aplastados. Vale anotar que cerca del $50 \%$ de neonatos mueren por aplastamiento en las primeras 48 a 72 horas después del nacimiento (García, Herradora \& Martínez, 2011).

De acuerdo con Peña (2011), la cerda posee períodos reproductivos y productivos más rápidos que las demás especies domésticas, los estrechos intervalos de tiempo entre partos, 142 a 149 días en zonas de producción comercial, con lactancia de 21 o 28 días, permiten tener 2,4 partos por año. El número de nacimientos obedece a la planeación de los períodos de producción, a la duración del parto (143 días) y de la lactancia (21 días), los cuales pueden cambiar (Sagarpa, 2016).

Martínez et al. (2014) mencionan que la etapa de destete, en la cadena de producción, es la más importante en la explotación de lechones; es por ello que el destete prematuro, a los 10 y 21 días de nacidos los lechones, permite que la cerda pueda generar una camada más cada dos años, esto es, cinco crías en vez de cuatro, o logra aumentar el número de cerdos con un $20 \%$ de cerdas menos.

Rendón del Águila, Gamba, Spilsbury y Herradora (2017) evaluaron el efecto del peso al parir, el tamaño de cría y lugar en la ubre, relacionando el crecimiento de los cerdos mientras lactan y engordan. Así mismo, Estévez (2016) analizó el proceso nutricional en la etapa de gestación y lactancia en un módulo de producción porcina y consideró inicialmente la nutrición de las cerdas reproductoras en las fases de preñez y lactancia, y la posibilidad del uso de dosis o productos no acostumbrados.

Para Rodríguez (2016), existen varios tipos de destete: ultraprecoz: se da en lechones menores de 21 días con un peso vivo entre 4 y $5 \mathrm{~kg}$; destete precoz: con edades entre 21 y 28 días con pesos que oscilan entre 6 y $8 \mathrm{~kg}$; destete funcional: se destetan a una edad de 42 días con pesos entre 8 y $12 \mathrm{~kg}$, y destete tradicional: se destetan a los 56-60 días con pesos entre 25 y $30 \mathrm{~kg}$.

Teniendo en cuenta todas las razones expuestas, en este trabajo se describe el tipo de jaulas o parideras tradicionales y tecnificadas que se manejan en la industria porcícola del departamento del Quindío, desde que el lechón nace hasta su destete a los 21 días aproximadamente. El objetivo de esta investigación es analizar los incrementos de peso de los lechones alojados en parideras tecnificadas y tradicionales durante su etapa de lactancia.

\section{MATERIALES Y MÉTODOS}

El estudio se llevó a cabo en las instalaciones de la Granja Experimental Bengala, de propiedad de la Universidad del Quindío, situada en la vereda Cruces del municipio de Filandia, a 2050 metros de altitud y una temperatura ambiental de 18 ${ }^{\circ} \mathrm{C}$. Tiene un área de 13 ha, aproximadamente, donde se desarrollan actividades 
agropecuarias (avicultura, ganadería, porcicultura, piscicultura, cunicultura, cultivos transitorios y permanentes).

\section{A. Etapas o fases}

La fase experimental se realizó en seis meses, se utilizaron 53 lechones de la raza landrace, estos fueron distribuidos en dos tratamientos: To: paridera o jaula tradicional (confinamiento), Figura 1, y T1: paridera o jaula tecnificada, Figura 2.

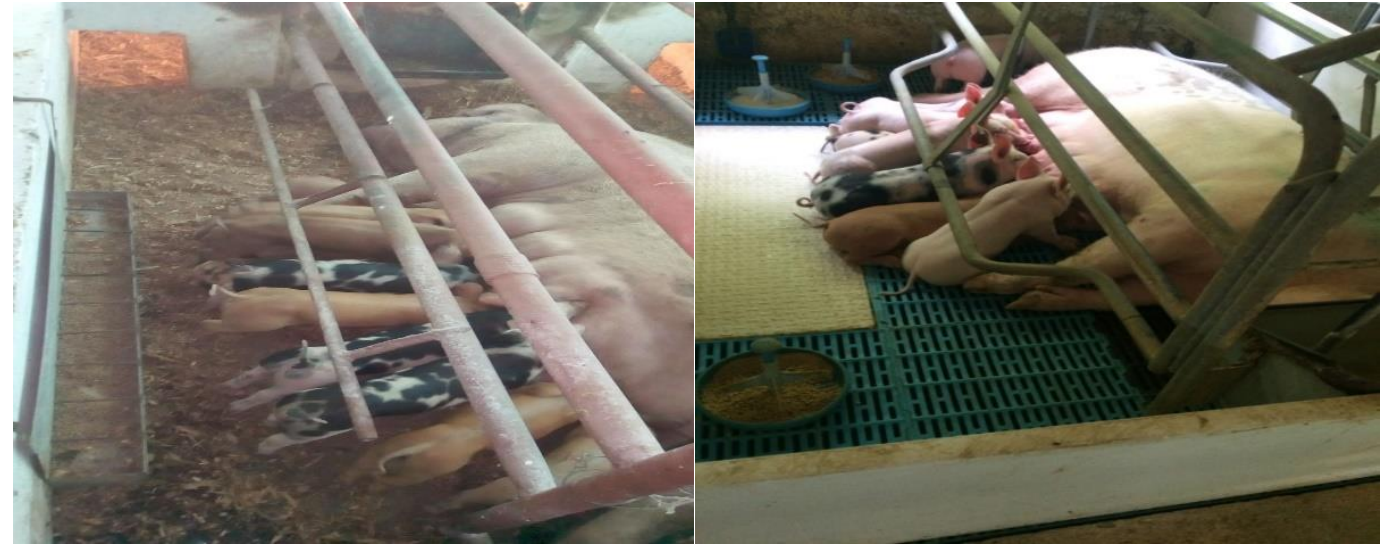

Fig. 1. Paridera tradicional.

Fig. 2. Paridera tecnificada.

El manejo para ambos tratamientos es semejante, con una previa esterilización de las jaulas (Ballina, 2010). Las cerdas gestantes parieron en el lugar propuesto como paritorio, adonde fueron llevadas cinco días antes de la fecha del parto. Cuando se estimaron la fecha y la hora aproximada de parto se prendieron las planchas térmicas o de calentamiento en las parideras tecnificadas (cuatro horas antes del parto); de esta manera, cuando los lechones nacieron permanecieron a una temperatura adecuada, la cual se estimó en $37^{\circ} \mathrm{C}$ para la primera semana y para la segunda semana se redujo a $32^{\circ} \mathrm{C}$, y a medida que los lechones fueron creciendo se ajustó la temperatura a $35^{\circ} \mathrm{C}$. Esto es necesario, porque al nacer el lechón en un lugar donde la temperatura esté por debajo de dicho nivel, tendrá que emplear energía adicional para mantener su calor corporal, de modo que dejará de crecer y, en el peor de los casos, consumirá aceleradamente sus reservas energéticas, situación que lo pondría en riesgo de muerte (Campbell, Crenshaw \& Polo, 2013; Quiles, 2004).

Se hicieron seguimientos a los lechones desde el primer día de nacidos hasta el destete a los 21 días (Casas \& Londoño, 2011), en las dos parideras (tradicional y tecnificada), las cuales tenían métodos de manejos muy similares. A los lechones se les tomó el peso inicial y cada ocho días se pesaron para saber con qué peso en promedio salían y, así, establecer cuál de los dos métodos era el más recomendable.

Para el análisis estadístico de las variables, peso al nacer, tiempo en días de la lactancia e incremento de peso predestete, se empleó un diseño de parcelas 
divididas con dos tratamientos, utilizando como base el programa estadístico Statgraphics XVII-X64.

\section{RESULTADOS Y DISCUSIÓN}

Las Tablas 1 y 2 muestran las medidas de las jaulas tecnificadas y tradicionales ubicadas en la Granja Bengala.

Tabla 1. Medias jaulas o parideras tradicionales.

\begin{tabular}{|l|l|}
\hline \multirow{2}{*}{ Jaula } & Ancho: $60 \mathrm{~cm}$ \\
\cline { 2 - 2 } & Largo: $2,20 \mathrm{~m}$ \\
\hline \multirow{2}{*}{ Cajón de maternidad } & Ancho: $1,40 \mathrm{~m}$ \\
\cline { 2 - 2 } & Largo: $2,20 \mathrm{~m}$ \\
\hline Cajón de los lechones & Ancho: $50 \mathrm{~cm}$ \\
\cline { 2 - 2 } & Largo: $1,40 \mathrm{~m}$ \\
\hline
\end{tabular}

Tabla 2. Medias jaulas o parideras tecnificadas.

\begin{tabular}{|l|l|}
\hline \multirow{3}{*}{ Cajón de maternidad } & Largo: $2,34 \mathrm{~m}$ \\
\cline { 2 - 2 } & Ancho: 1,36 \\
\cline { 2 - 2 } & Alto: $50 \mathrm{~cm}$ \\
\hline \multirow{4}{*}{ Jaula paridera } & Parte delantera: $62 \mathrm{~cm}$ \\
\cline { 2 - 2 } & Parte trasera: $64 \mathrm{~cm}$ \\
\cline { 2 - 2 } & Largo: $2 \mathrm{~m}$ \\
\cline { 2 - 2 } & Ancho: $63 \mathrm{~cm}$ \\
\cline { 2 - 2 } & Alto: $95 \mathrm{~cm}$ \\
\hline Piso jaula de maternidad & Ancho: $1,15 \mathrm{~m}$ \\
\cline { 2 - 2 } & Largo: $1,82 \mathrm{~m}$ \\
\hline Plancha térmica & Ancho: $1,19 \mathrm{~m}$ \\
\cline { 2 - 2 } & Largo: $36 \mathrm{~cm}$ \\
\hline
\end{tabular}

Los galpones o piaras estaban bien ventilados, con techos altos, jaulas para alojar individualmente a cada cerda, esto con el fin de tener un estricto control durante todo el periodo de gestación (Castellanos, 2012).

En la Figura 3 se observa el peso promedio a los 21 días de los lechones en la fase de lactancia en parideras tradicionales y tecnificas. 


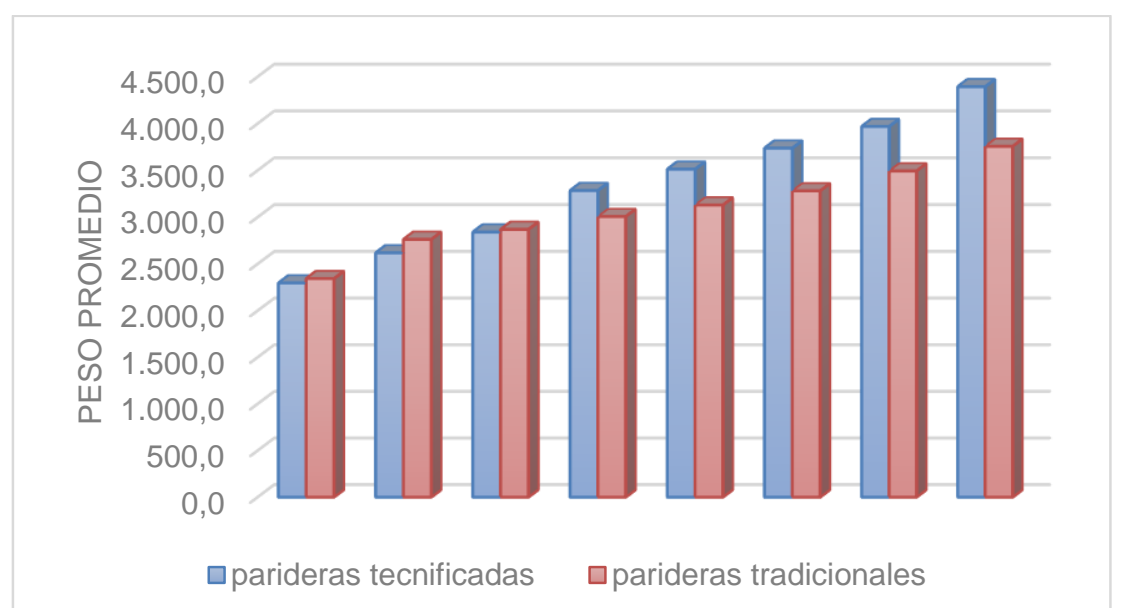

Fig. 3. Peso promedio de lechones en jaulas tecnificas y tradiciones.

Los pesos registrados al nacer los lechones en parideras o jaulas tradicionales y tecnificadas concuerdan con lo expuesto por Rendón del Águila et al. (2017), y Rehfeldt, Hartung y Kuhn (2010), quienes afirman que el peso promedio de los lechones al nacer es de $1274 \mathrm{~g}$ y el máximo es de $1562 \mathrm{~g}$. En cuanto al peso en el destete, Roppa (2003) asegura que el peso ideal al destete está entre 6,0 y 7,2 kg, los cuales son muy parecidos a los promedios de los pesos de los lechones en jaulas tradicionales y tecnificadas.

La duración del periodo de lactancia de los lechones está íntimamente relacionada con el peso de los lechones al momento del destete. Los datos obtenidos en este estudio son menores que los referidos por Beltrán, Velásquez y Pérez (2011), quienes indicaron que para explotaciones comerciales porcícolas de Cundinamarca el peso es de $5,5 \mathrm{~kg}$, de todos los lechones con 21 días de lactancia. García (2011) confirmó que, con 28 días de lactancia, los lechones alcanzan un peso de 7,8 kg. Igualmente, Díaz et al. (2011) reportaron un peso promedio al destete de $6.09 \mathrm{~kg}$ (22 días) por lechón. La disminución en la fase de lactancia se ha obtenido con el mejoramiento de las técnicas de sanidad, nutrición y manejo, que buscan beneficiar a la cerda hasta que adquiera el máximo de producción de leche, lo que permite aumentar la producción de la piara (Díaz et al., 2011; Cavalcante-Neto et al., 2009).

Este proyecto se realizó con el destete precoz (21 días), en el cual se evidencia que los pesos al destete oscilan entre 5 y $7 \mathrm{~kg}$ dependiendo del sistema (tradicional o tecnificado).

En cuanto a la identificación del mejor método (tratamiento) para la ganancia de peso, se pudo establer mediante el análisis de varianza que ningún valor-P es menor que 0,05 ; por tanto, ninguno de los factores tiene un efecto estadísticamente significativo sobre el peso con un $95 \%$ de nivel de confianza (Figura 4). 


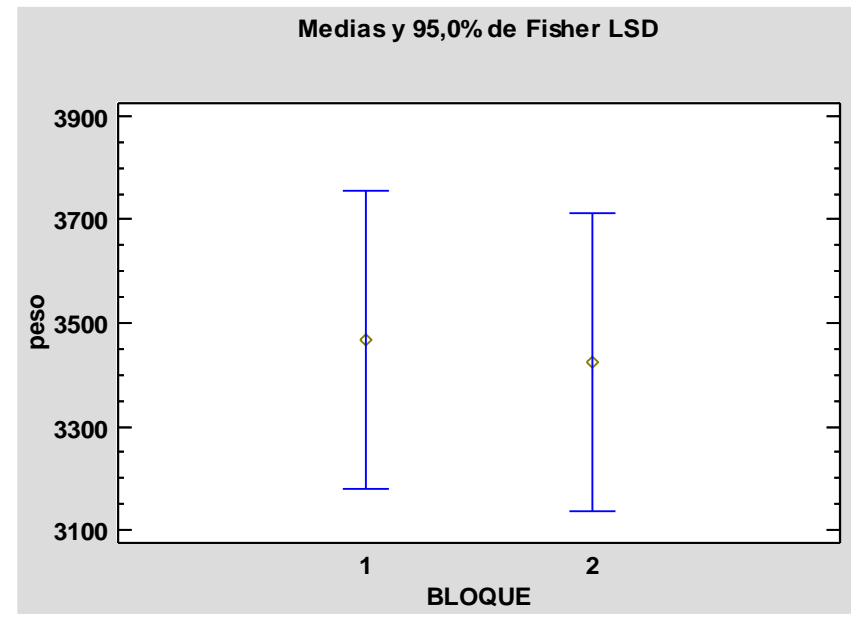

Fig. 4. El método de diferencia mínima significativa (LSD) de Fisher.

Con los datos tomados de la literatura y los obtenidos durante el desarrollo del proyecto se demuestra que los pesos resultantes varían según el sistema utilizado (tradicional o tecnificado), lo que significa que un lechón está teniendo una ganancia de peso de 150 a $200 \mathrm{~g}$ por día.

\section{CONCLUSIONES}

El promedio general desde el nacimiento hasta el destete (21días) en parideras tecnificadas está en $6400 \mathrm{~g} / \mathrm{lechón}$, lo que indica que el aumento semanal de los lechones en jaulas tecnificadas se encuentra en un promedio de $1300 \mathrm{~g}$; en cambio, el promedio general de los lechones al destete (21 días) en jaulas tradicionales está entre $5450 \mathrm{~g} / \mathrm{lechón}$, lo cual muestra que el promedio semanal de los lechones en jaulas tradicionales es de $1200 \mathrm{~g}$.

\section{REFERENCIAS}

Asociación Colombiana de Porcicultores -Porcicol-. (2009). Buenas prácticas porcinas. Recuperado de http://www.porcicol.org.co/tecnica/tecnica.php

Ballina, G., \& Bencomo, A. (2010). Manejo sanitario eficiente de los cerdos. Organización de las Naciones Unidas para la Agricultura y la Alimentación (FAO). Programa Especial para la Seguridad Alimentaria (PESA). Instituto Nicaragüense de Tecnología Agropecuaria (INTA). Recuperado de http://www.fao.org/3/a-as542s.pdf

Beltrán, G. E. (2010). Factores que afectan los procesos de alimentación y nutrición del cerdo neonato y destetado. Los Porcicultores y su Entorno, 13(75), 38-44.

Beltrán, R. G., Velázquez, E. H., \& Pérez, J. E. (2011). Prácticas alimenticias en lechones en lactación y postdestete (parte I). Los Porcicultores y su Entorno, 13(76), 154-160.

Brunori, J., Rodríguez, F. M., \& Figueroa, M. E. (2012). Buenas prácticas pecuarias para la producción y comercialización porción familiar, (1. ㄹ ed.). Buenos Aires: FAO.

Campbell, J., Crenshaw, J., \& Polo, J. (2013). The Biological Stress of Early Weaned Piglets. Journal of Animal Science and Biotechnology, 4(1), 19.

Casas, A., \& Londoño, O. D. (2011). Propuesta para un sistema de producción porcícola en lbagué. Recuperado de http://repository.lasalle.edu.co/handle/10185/5423

Ciencia y Agricultura (Cien. Agri.) Vol. 16 (3). L-ISSN 0122-8420. elSSN 2539-0899.

Septiembre-Diciembre 2019, pp. 7-16. Tunja (Boyacá) - Colombia. DOI:

https://doi.org/10.19053/01228420.v16.n3.2019.9513 
María Johana García-Aguirre; Rigoberto Villa-Ramírez; Jaime Hurtado-Villegas

Castellanos, G. Edi (2012). Diseño Óptimo De Una Granja Porcina. Recuperado de https://es.scribd.com/doc/143706313/Diseno-optimo-de-una-granja-porcina-pdf

Cavalcante-Neto, A., Lui, J. F., Sarmento, J. L. R., Ribeiro, M.N., Monteiro, J.M.C, Fonseca, C., \& Tonhati, H. (2009). Efeitos genéticos e não-genéticos sobre o intervalo de parto em fêmeas suínas no Sudeste do Brasil. Arquivo Brasileiro de Medicina Veterinária e Zootecnia, 61, 280-285. https://doi.org/10.1590/s0102-09352009000100040.

Díaz, C., Rodríguez, N., Vera, V., Ramírez, G., Casas, G., \& Mogollón, J. (2011). Caracterización de los sistemas de producción porcina en las principales regiones porcícolas colombianas. Revista Colombiana de Ciencia Pecuaria, 24, 131-144.

Estévez, J. A. (2016). Manejo alimentario durante la gestación y lactancia en una unidad integral de producción porcina. Estudio de caso. Rev. prod. anim., 28(2), 1-11.

García, J. S., Herradora, M. A., \& Martínez, R. G. (2011). Efecto del número de parto de la cerda, la caseta de parición, el tamaño de la camada y el peso al nacer en las principales causas de mortalidad en lechones. Revista Mexicana de Ciencias Pecuarias, 2(4), 403-414.

Manteca, X. (2011). Bienestar animal en explotaciones de porcino. Revista Colombiana de Ciencias Pecuarias, 24(3), 303-305.

Martínez, J., Amorin, A., Faria, D., Nakagi, V., Sartori, M., \& Marques, M. (2014). Palatabilizantes em dietas de leitões recém-desmamados. SCieloBrazil. Arq. Bras. Med. Vet. Zootec., 66(4). https://doi.org/10.1590/1678-6327.

Peña, D. (2011). Guía de manejo para la cría de cerdas de reemplazo con inseminación artificial en trópico alto. (Trabajo de grado). Corporación Universitaria Lasallista, Caldas.

Piñeiro, C., \& Montalvo, G. (2015). Guía de mejores técnicas disponibles para el sector porcícola en Colombia. Colombia: Dpto. de Ingeniería Ambiental y Proyectos Pig CHAMP Pro Europa, Fondo Nacional de la Porcicultura.

Quiles, A. (2004). Factores que inciden en la mortalidad neonatal en los lechones. Producción animal. Murcia, España: Departamento de Producción Animal, Facultad de Veterinaria, Universidad de Murcia.

Rehfeld, C. T., Hartung, M., \& Kuhn, G. (2010). El peso al nacimiento de los cerdos influye en la calidad de la canal y la carne. https://www.portalveterinaria.com/articoli/articulos/7519/el-peso-al-nacimiento-delos-cerdos-influye-en-la-calidad-de-la-canal-y-la-carne.html.

Rendón del Águila, J. U., Martínez, R. G., Lozano, M. A., Spilsburyb, A., \& Herradora, M. (2017). Efecto del peso al nacer, tamaño de camada y posición en la ubre sobre el crecimiento de cerdos durante la lactancia y engorda. Revista Mexicana de Ciencias Pecuarias, 8(1), 75-81. https://doi.org/10.22319/rmcp.v8i1.4317.

Rodríguez, D. P. (2016). Consideraciones sobre el destete en lechones. (Monografía). Universidad de Ciencias Aplicadas y Ambientales UDCA Vicerrectoría de Investigaciones Facultad de Ciencias Pecuarias, Bogotá.

Roppa, L. (2003). La nutrición y la alimentación de las hembras reproductoras en gestación. Recuperado de http://www.engormix.com/MA-porcicultura/articulos/nutricion-alimentación-hembras-reproductorast484/p0.htm.

Sagarpa (2016). Sistema de producción porcina. Recuperado de http://www.sagarpa.gob.mx/ganaderia/Paginas/Inicio.aspx.

Ciencia y Agricultura (Cien. Agri.) Vol. 16 (3). L-ISSN 0122-8420. elSSN 2539-0899.

Septiembre-Diciembre 2019, pp. 7-16. Tunja (Boyacá) - Colombia. DOI:

https://doi.org/10.19053/01228420.v16.n3.2019.9513 